\title{
Risk Factors for Cognitive Impairment in High-Grade Glioma Patients Treated with Postoperative Radiochemotherapy
}

\author{
Oiang Wang, MS 1,2 \\ Fengxia Xiao, $\mathrm{MD}, \mathrm{PhD}{ }^{3}$ \\ Fei Oi, MS² \\ Xiaopeng Song, MS ${ }^{4}$ \\ Yonghua Yu, MD, PhD'
}

\begin{abstract}
${ }^{1}$ Department of Radiation Oncology, Shandong Cancer Hospital Affiliated to Shandong University, Jinan, ${ }^{2}$ Department of Oncology, Laiwu Central Hospital of Xinwen Mining Group, Jinan, ${ }^{3}$ Department of Radiation Oncology, Qilu Hospital of Shandong University, Jinan, ${ }^{4}$ Department of Oncology, No. 88 Hospital of People's Liberation Army, Tai'an, China
\end{abstract}

\begin{abstract}
Purpose
Fractionated radiotherapy as well as concomitant and adjuvant chemotherapy such as temozolomide for postoperative high-grade glioma (HGG) patients improves progressionfree survival and overall survival. Multiple factors such as chemotherapy, radiotherapy, tumor grade, residual tumor volume, and genetic modifications might play a role in the formation of cognitive impairment. The risk factors of cognitive impairment in postoperative patients with HGG receiving radiotherapy and chemotherapy remains a concern in this population. The purpose of this study was to identify risk factors for cognitive impairment in patients of postoperative HGG.
\end{abstract}

\section{Materials and Methods}

A total of 229 patients with HGG who underwent surgery were analyzed. Cognitive impairment was defined as a decrease of Cognitive Assessment Montreal (MoCA)'s score in at least two cognitive domains or any MoCA's score of less than 26 points at the time of study compared with baseline level. Multiple potential risk factors including methylated status of the $0^{6}$-methylguanine-DNA methyltransferase (MGMT) promoter, glioma World Health Organization (WHO) grade, residual tumor volume, education, and sex were analyzed. Cox univariate and multivariate regression analysis was used to detect the significant risk factors for cognitive impairment.

\section{Results}

At the end of follow-up among the 229 patients, 147 patients (67\%) developed cognitive impairment. 82 patients (36\%) remained in normal cognitive condition. In multivariate analysis, unmethylated MGMT promoter (hazard ratio [HR], 1.679; 95\% confidence interval [Cl], 1.212 to 2.326; $p=0.002$ ), glioblastoma ( $\mathrm{HR}, 1.550 ; 95 \% \mathrm{Cl}, 1.117$ to 2.149; $\mathrm{p}=0.009$ ), and residual tumor volume $>5.58 \mathrm{~cm}^{3}(\mathrm{HR}, 1.454 ; 95 \% \mathrm{Cl}, 1.047$ to $2.020 ; \mathrm{p}=0.026)$ were independent risk factors for cognitive impairment.

\section{Conclusion}

Methylated status of the MGMT promoter, glioma WHO grade, and residual tumor volume might be risk factors for the cognitive impairment in postoperative patients with HGG.

\section{Key words}

Risk factors, Cognitive dysfunction, High-grade glioma, Radiotherapy, Chemotherapy

\section{Introduction}

High-grade glioma (HGG) is the most common primary brain tumor in the adult central nervous system (CNS), the incidence of which is $27 \%$ of the primary CNS tumors and
$80 \%$ of the malignant primary CNS tumors [1]. According to World Health Organization (WHO) classification, glioma is divided into grade I-IV, of which grade I and II for low-grade glioma, grade III and IV for HGG, also known as malignant glioma.

With the progress of surgery, radiotherapy, and chemothe- 
rapy, the 2-year survival rate of patients with malignant primary CNS tumors can be increased to $44.1 \%$ [1]. With the prolongation of survival, more attention was paid on cognitive dysfunction of patients with HGG and the decline of life quality.

Tumor itself can lead to cognitive dysfunction. Rapid growth of HGG with high intracranial pressure and neurological dysfunction as the main manifestations could conceal the relative occult cognitive dysfunction [2]. But a detailed examination of 139 patients with different brain tumors found cognitive impairment in 126 patients (91\%) [3]. This has attracted our attention on the study of cognitive dysfunction which might have been underestimated in current knowledge.

Radiotherapy has been considered to be the main cause of cognitive impairment [4]. But in recent years, radiotherapy was not considered as the main reason since patients only receive a small region of radiation [5]. Most of the prospective studies less than 5 years of follow-up showed that radiotherapy did not lead to a decline in cognitive function, or was not the main cause of cognitive dysfunction [6,7]. Only one prospective study suggested that radiation might have a detrimental effect on cognitive function, but the patients were followed for more than 10 years [8].

Chemotherapy has a negative effect on cognitive function. Temozolomide (TMZ) is a new type of alkylating agent, with outstanding characteristics including small molecule size, lipid solubility, high degree of biological utilization. After entering the CNS and transforming to antitumor bioactive substances, it could generate a synergistic effect with radiotherapy $[9,10]$. At present, concurrent radiotherapy and chemotherapy is commonly used in HGG. Retrospective studies have shown that older patients may be associated with cognitive impairment [11].

Cognitive Assessment Montreal (MoCA) is a simple, rapid and convenient tool for screening mild cognitive impairment. It has higher sensitivity and more comprehensive coverage compared with the traditional cognitive screening scale, the Mini-Mental-State Examination (MMSE), which has been widely used [12-14]. The latest version of MoCA includes the following areas: short term memory and delayed recall, visual spatial ability, executive ability, attention, computational power, working memory, language and orientation ability. The total score is 30 points, and the required time for completion is about 10 minutes. Cognitive impairment was defined as a decrease of MoCA's score in at least two cognitive domains [15] or any MoCA's score of less than 26 points at the time of study compared with baseline level before radiotherapy. For those who received education of less than or equal to 12 years, the MoCA's score would be deducted for 1 point to correct bias of cultural degree.

Clinical and molecular biological indicators such as glioma

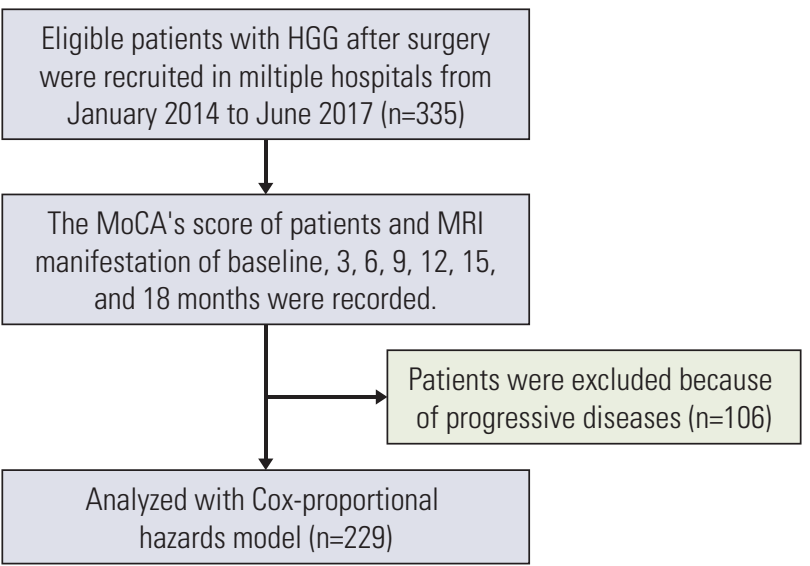

Fig. 1. Flowchart of the study. HGG, high-grade glioma; MoCA, Cognitive Assessment Montreal; MRI, magnetic resonance imaging.

location, grading, residual tumor volume, sex, Karnofsky Performance Status (KPS), peritumoral edema, and $\mathrm{O}^{6}$-methylguanine-DNA methyltransferase (MGMT) promoter methylation status might be risk factors for cognitive impairment in postoperative patients with HGG. The aim of this study was to screen for significant risk factors for cognitive impairment in patients with HGG resection.

\section{Materials and Methods}

The flowchart of our study is shown in Fig. 1.

\section{Patients}

HGG patients after surgery were recruited in multiple hospitals. The recruiting time period was from January 2014 to June 2017. Inclusion criteria included: reception of adjuvant radiotherapy and chemotherapy after HGG resection, age $>18$ years, signature of informed consent. Exclusion criteria included: inability of reading or answering the questionnaire, KPS $<70$, life expectancy $<3$ months, other CNS tumors, other diseases that can cause cognitive impairment (brain trauma, stroke), unsuitability for participation for any other reason.

\section{Radiotherapy and chemotherapy protocol with TMZ}

For patients with newly resected HGG, standard treatment included fractionated external beam radiation therapy with 
concomitant administration of TMZ followed by up to six cycles of adjuvant TMZ (combined chemotherapy and radiotherapy [CCRT]). The detailed description of radiotherapy and TMZ treatment was referred to National Comprehensive Cancer Network clinical practice guidelines in Oncology: Central Nervous System Cancers (2014.V1) and the reference [16]. HGG could be a histological grade 3 anaplastic glioma (AG) or a grade 4 glioblastoma (GBM). For AG, T2-weighted magnetic resonance imaging (MRI) sequences and the region of contrast enhancement in T1-weighted MRI sequences are considered for the delineation of the gross tumor volume, and an isotropic expansion of 15 to $20 \mathrm{~mm}$ is recommended for the clinical target volume (CTV). For GBM, the Radiation Therapy Oncology Group favors a two-step technique, with an initial phase (CTV1) including any T2 hyperintensity area (edema) plus a $20 \mathrm{~mm}$ margin treated with up to $46 \mathrm{~Gy}$ in 23 fractions, followed by a reduction in CTV2 to the contrast enhancement region in $\mathrm{T} 1$ with an additional $25 \mathrm{~mm}$ margin. A total dose of $60 \mathrm{~Gy}$ in 30 fractions is usually delivered for HGG. Based on the fusion of MRI enhanced scan and computed tomography plain scan image, the radiotherapy target area delineation design is performed. Irradiation was delivered to the patients by a 6-MV X-ray linear accelerator. All the patients received conformal external beam radiotherapy administered in a daily dosage of 2 Gy for 5 days per week, achieving a total dose of $60 \mathrm{~Gy}$. All the patients received concurrent TMZ at a dose of $75 \mathrm{mg} / \mathrm{m}^{2} /$ day orally. Additionally, adjuvant six cycles of TMZ (150-200 mg/ $\mathrm{m}^{2}$ for 5 days) were given in every 28 days.

\section{Protocol for evaluating risk factors}

Cognitive impairment was defined as a decrease in the score of at least two cognitive domains or MoCA's score $<26$ points compared with baseline (before combined chemotherapy and radiotherapy). For those who received education of less than or equal to 12 years, the MoCA's score would be deducted for 1 point to correct cultural degree bias. Key eval- uations were made at baseline (after surgery and before radiotherapy and chemotherapy) and at 3, 6, 9, 12, 15, and 18 months after the end of radiotherapy. The content of evaluations included MoCA's score and the analysis of concurrent brain enhanced MRI examination. Follow-up was discontinued at the time of tumor progression. Brain MRI examination was used to assess recurrence or progression diseases.

\section{Statistical analysis}

The incidence of cognitive impairment at different time points of treatment was calculated using the Kaplan-Meier method. The potential risk factors considered in the analysis were age, KPS, laterality, sex, residual tumor volume, education, MGMT promoter methylation status, and edema. All risk factors except residual tumor volume were included as categorical variables in the model. The cutoff value of the residual tumor volume determined by the receiver operating characteristic (ROC) curve is used as its categorical variable. Univariate Cox proportional hazard models were used to evaluate risk factors of cognitive impairment in postoperative patients with HGG. The p-value of less than 0.05 is considered to be statistically significant. The independent risk factors of the cognitive impairment were screened by multivariate Cox proportional hazard models. The discrimination ability of the prediction model is indicated by the most commonly used area under the ROC curve (AUC), also known as $\mathrm{C}$-statistics. The larger the AUC, the better the discriminative ability of the predictive model. Generally, AUC $<0.6$ indicates that the discrimination degree is low, AUC 0.6-0.75 indicates that the model has a certain distinguishing ability, and AUC $>0.75$ implies that the distinguishing ability is better. The Hosmer-Lemefitting test is used to evaluate the calibration ability of the prediction model. All analyses were performed using SPSS ver. 15.0 (SPSS Inc., Chicago, IL).

Table 1. Characteristics of the study population

\begin{tabular}{lccc} 
Time point & Cognitive impairment & Disease progression & Eligible patients \\
1 (before treatment) & 0 & - & 335 \\
$2(3 \mathrm{mo})$ & 20 & 12 & 303 \\
$3(6 \mathrm{mo})$ & 15 & 16 & 272 \\
$4(9 \mathrm{mo})$ & 56 & 24 & 192 \\
$5(12 \mathrm{mo})$ & 27 & 26 & 139 \\
$6(15 \mathrm{mo})$ & 10 & 17 & 112 \\
$7(18 \mathrm{mo})$ & 19 & 11 & 82 \\
Total & 147 & 106 & \\
\hline
\end{tabular}


Table 2. Demographic, clinical, and molecular characteristics of patients.

\begin{tabular}{|c|c|}
\hline Clinical characteristic & No. of patients $(\%)$ \\
\hline \multicolumn{2}{|l|}{ Sex } \\
\hline Male & $135(59.0)$ \\
\hline Female & $94(41.0)$ \\
\hline \multicolumn{2}{|l|}{ Age (yr) } \\
\hline$<60$ & $118(51.5)$ \\
\hline$\geq 60$ & $111(48.5)$ \\
\hline \multicolumn{2}{|l|}{ Laterality } \\
\hline Left & $124(54.1)$ \\
\hline Right & $105(45.9)$ \\
\hline \multicolumn{2}{|c|}{ Mean residual tumor volume $\left.{ }^{a}\right)\left(\mathrm{cm}^{3}\right)$} \\
\hline$\leq 5.58$ & $92(40.2)$ \\
\hline$>5.58$ & $137(59.8)$ \\
\hline \multicolumn{2}{|l|}{ WHO grade } \\
\hline Anaplastic astrocytoma & $88(38.4)$ \\
\hline Glioblastoma & $141(60.5)$ \\
\hline \multicolumn{2}{|l|}{ KPS (\%) } \\
\hline $70-80$ & $78(34.1)$ \\
\hline $80-90$ & $102(44.5)$ \\
\hline $90-100$ & $49(21.4)$ \\
\hline \multicolumn{2}{|l|}{ Edema } \\
\hline Yes & $104(45.4)$ \\
\hline No & $125(54.6)$ \\
\hline \multicolumn{2}{|l|}{ Education (yr) } \\
\hline$<12$ & $127(55.5)$ \\
\hline$\geq 12$ & $102(44.5)$ \\
\hline \multicolumn{2}{|c|}{ MGMT promoter methylation status ${ }^{\text {b) }}$} \\
\hline Positive & $131(57.2)$ \\
\hline Negative & $98(42.8)$ \\
\hline \multicolumn{2}{|l|}{ Location } \\
\hline Frontal lobe & 34 (14.8) \\
\hline Temporal lobe & $125(54.6)$ \\
\hline Parietal lobe & $26(11.4)$ \\
\hline Occipital lobe & 44 (19.2) \\
\hline
\end{tabular}

WHO, World Health Organization; KPS, Karnofsky Performance Status; MGMT, $\mathrm{O}^{6}$-methylguanine-DNA methyltransferase; ROC, receiver operating characteristic; AUC, area under the curve; $\mathrm{CI}$, confidence interval. ${ }^{a}$ Residual tumor volume: determined by $\mathrm{T} 1$ enhanced magnetic resonance imaging. The ROC analysis showed that the optimal cutoff value for glioma volume was $5.58 \mathrm{~cm}^{3}$ (AUC, 0.778; 95\% CI, 0.714-0.843; sensitivity, 0.823 ; specificity, 0.645; Yoden index, 0.469), b) MGMT promoter methylation status was determined by pyrosequencing using cutoff value of $>5 \%$ positivity of methylated alleles.

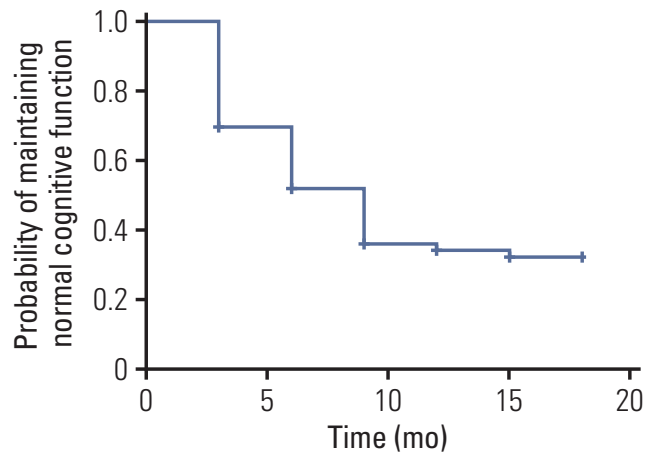

Fig. 2. The incidence of cognitive impairment in the cohort $(\mathrm{n}=229)$ presented in a survival plot.

\section{Ethical statement}

The research followed the Helsinki declaration (guidelines Helsinki). The study was approved by the Institutional Review Board of Laiwu Central Hospital, Xinwen Mining Group (IRB No. 201401016). All patients signed the informed consent.

\section{Results}

Table 1 provides information on the number of patients eligible for analysis at each follow-up timepoint.

Table 2 shows the characteristics of the study population. Among the respondents, $60.5 \%$ were $\mathrm{WHO}$ grade IV and $57.2 \%$ had methylated MGMT promoter.

Fig. 2 shows the incidence of cognitive impairment at different time points.

At the end of follow-up, 147 patients (64.2\%) developed cognitive impairment and 82 patients $(35.8 \%)$ did not. The median follow-up duration regarding cognitive function was 9.0 months (95\% confidence interval [CI], 7.85 to 10.15 ) and the incidence of cognitive impairment curve is presented in Fig. 2. MoCA scores of baseline (before radiotherapy and chemotherapy), $3,6,9,15$, and 18 months were $26.86 \pm 1.00$, $26.49 \pm 2.15,26.80 \pm 1.64,26.29 \pm 1.85,26.34 \pm 2.92,26.70 \pm 2.13$, and $26.31 \pm 2.85$, respectively. One-way ANOVA analysis for MoCA score showed that there were significant differences between each follow-up timepoint $(\mathrm{F}=2.33, \mathrm{p}=0.03)$, among which, significant difference was found between the 9 months and baseline group, no differences were found between other groups.

The incidence of cognitive impairment in the cohort $(\mathrm{n}=229)$ presented in a survival plot (Fig. 2). 
Table 3. Results of Cox univariate and multivariate regression analysis

\begin{tabular}{|c|c|c|c|c|c|}
\hline & \multicolumn{2}{|c|}{ Univariate analysis } & \multicolumn{2}{|c|}{ Multivariate analysis } & \multirow{2}{*}{$\begin{array}{c}95 \% \text { CI for } \\
\text { Multivariate HR }\end{array}$} \\
\hline & HR & p-value & HR & p-value & \\
\hline Sex & 0.730 & 0.067 & 1.212 & 0.272 & 0.86-1.709 \\
\hline Edema & 0.965 & 0.831 & - & - & - \\
\hline Education & 0.742 & 0.080 & 1.205 & 0.276 & $0.862-1.686$ \\
\hline KPS & 0.948 & 0.644 & - & - & - \\
\hline Age & 0.762 & 0.104 & - & - & - \\
\hline MGMT & 1.657 & 0.002 & 1.813 & 0.001 & $1.297-2.535$ \\
\hline Laterality & 0.807 & 0.201 & - & - & - \\
\hline Volume & 1.489 & 0.017 & 1.454 & 0.021 & $1.06-2.044$ \\
\hline Grade & 1.501 & 0.015 & 1.449 & 0.029 & $1.039-2.02$ \\
\hline Lobe & 1.714 & 0.043 & 1.433 & 0.271 & $0.755-2.718$ \\
\hline
\end{tabular}

HR, hazard ratio; CI, confidence intervla; KPS, Karnofsky Performance Status; MGMT, O'-methylguanine-DNA methyltransferase.

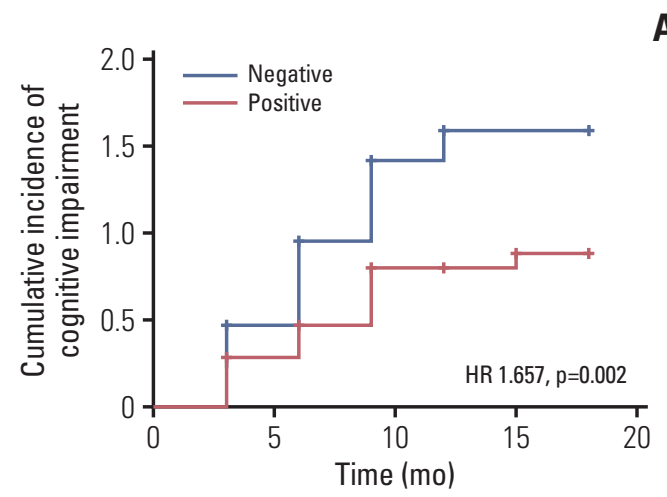

A

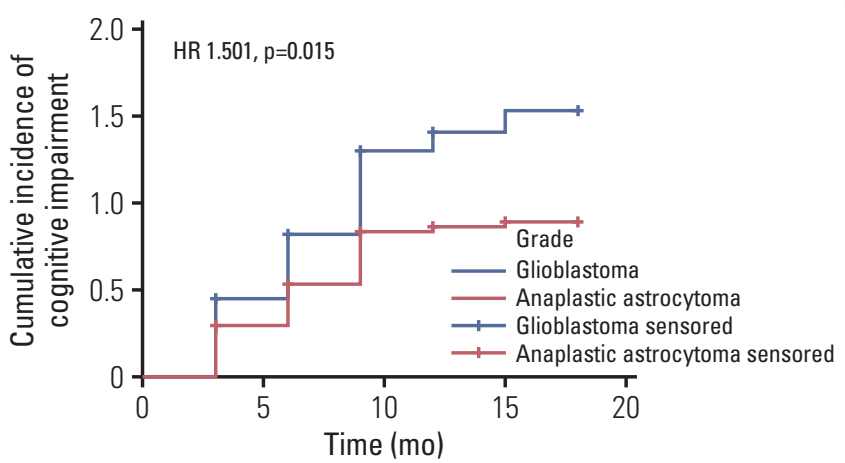

B

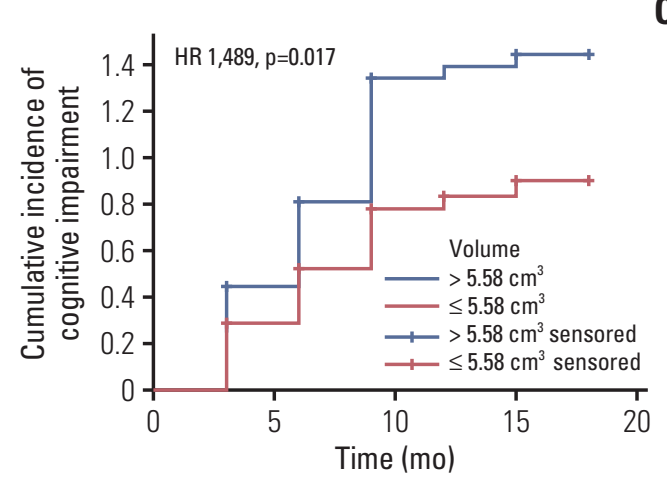

Fig. 3. Cognitive impairment curves for the independent predictors. (A) $\mathrm{O}^{6}$-methylguanine-DNA methyltransferase (MGMT) promoter methylation status of high-grade glioma (HGG). (B) HGG. (C) Residual tumor volume of HGG.

We evaluated the established risk factors $(\mathrm{p}<0.1)$ of cognitive impairment in a Cox multivariate survival analysis. The results are presented in Table 3 and Fig. 3A-C. In multivariate analysis that included MGMT promoter methylation status, WHO glioma grade, residual tumor volume, education, and sex, we found three independent risk factors for cognitive impairment: positive MGMT promoter methylation (hazard ratio [HR], 1.679; 95\% CI, 1.212 to 2.326 ; $\mathrm{p}=0.002$ ) 
Table 4. Delivered dose to the bilateral hippocampi and the whole brain

\begin{tabular}{lccccc} 
Group & No. & $\begin{array}{c}\text { Maximum dose } \\
\text { for BH (Gy) }\end{array}$ & $\begin{array}{c}\text { Mean dose } \\
\text { for BH (Gy) }\end{array}$ & $\begin{array}{c}\text { Maximum dose } \\
\text { for WB (Gy) }\end{array}$ & $\begin{array}{c}\text { Mean dose } \\
\text { for WB (Gy) }\end{array}$ \\
CI & 147 & $39.28 \pm 8.14$ & $31.85 \pm 9.13$ & $57.14 \pm 3.79$ & $30.43 \pm 3.65$ \\
NCI & 82 & $41.00 \pm 8.61$ & $33.62 \pm 9.02$ & $58.01 \pm 4.33$ & $31.20 \pm 3.97$ \\
t & & -1.49 & -1.53 & -1.51 & -1.45 \\
p-value & 0.139 & 0.127 & 0.133 & 0.149 \\
\hline
\end{tabular}

$\mathrm{BH}$, bilateral hippocampi; WB, whole brain; $\mathrm{CI}$, cognitive impairment; NCI, no cognitive impairment.

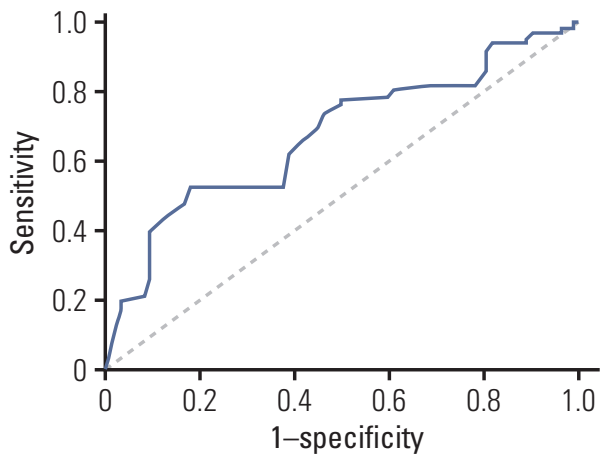

Fig. 4. Discrimination for risk prediction model of cognitive impairment by receiver operating characteristic curve.

(Fig. 3A), GBM (HR, 1.550; 95\% CI, 1.117 to 2.149; $\mathrm{p}=0.009$ ) (Fig. 3B), residual tumor volume $>5.58 \mathrm{~cm}^{3}(\mathrm{HR}, 1.454 ; 95 \%$ CI, 1.047 to 2.020; $\mathrm{p}=0.026$ ) (Fig. 3C).

Cognitive impairment curves for the independent predictors presented in Table 3 including MGMT promoter methylation status (Fig. 3A), glioma grade (Fig. 3B), residual tumor volume (Fig. 3C).

We further analyzed the radiotherapy dose (Gy, $\mathrm{x} \pm \mathrm{s})$ delivered to the bilateral hippocampi and the whole brain in cognitive impairment group and no cognitive impairment group. Using $t$ test analysis for independent samples by SPSS statistical software, no significant differences were found for the delivered dose of bilateral hippocampi and the whole brain between the two groups. Accordingly, the delivered dose for bilateral hippocampi and the whole brain was excluded as a confounding factor in the current study (Table 4).

The discrimination ability of the risk prediction model was measured using C-statistics and was found to be 0.671 (95\% CI, 0.600 to 0.742 ). Figs. 4 and 5 show the discrimination and calibration plots for the cognitive impairment risk prediction model. The Hosmer-Lemeshow type chi-square value was 4.085 ( $\mathrm{p}=0.665)$.

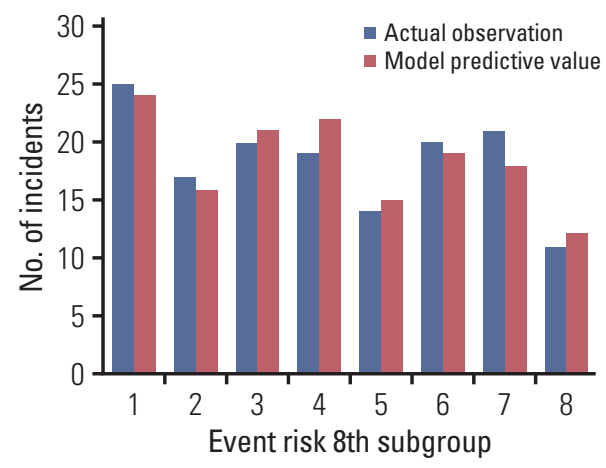

Fig. 5. Calibration of cognitive impairment risk model.

\section{Discussion}

In recent years, CCRT followed by adjuvant TMZ is the standard treatment for postoperative HGG patients. The regimen had more favorable survival rates than those who did not undergo this treatment and tolerable toxicity $[17,18]$. The application of molecular biology for precision medicine can accurately reflect the molecular biological characteristics and clinical treatment effects and prognosis of glioma $[19,20]$. Multiple molecular biology indicators and gene factors of glioma were reported to be related to neurocognitive function [21]. For example, the IDH1 gene mutation was considered to contribute to cognitive impairment in malignant astrocytoma [22].

The current study prospectively examined risk factors for cognitive impairment in postoperative patients with HGG after radiotherapy. We firstly found that MGMT promoter methylation status was an independent risk factor. MGMT is a repair enzyme that removes promutagenic $\mathrm{O}^{6}$-methylguanine adducts in DNA. The methylation of MGMT promoter may affect MGMT expression and activity. The DNA repair protein MGMT is known to play a role in affecting sensitivity to TMZ [23] and is significantly associated with sur- 
vival [24] and is an independent risk factor for prognosis [25]. Negative MGMT promoter methylation status as higher risk of cognitive impairment than positive status. Considering the large number oncogenic or promutagenic molecules that play a role in glioma development and progression, more potential risk factors for cognitive impairment in postoperative patients with HGG need to be further explored in the context of precision medicine [26].

Another independent risk factor for cognitive impairment is WHO histological grade of glioma. Previous findings demonstrated different levels of impairment in the executive and memory domains inpatients with low- and high-grade glioma [27]. Compared with patients with grade II or III left temporal lobe glioma, patients with grade IV glioma exhibit greater difficulty with verbal learning, processing speed, executive functioning, and language [28]. The study for longterm effects of radiotherapy on cognitive function in adult low-grade glioma patients reported that the group receiving postoperative irradiation performed significantly worse than the group without radiotherapy. This difference was not attributed to histologic diagnosis; location, extent of removal, or progression of the tumor [29]. Our study found that grade IV glioma has a higher risk of cognitive impairment than that of grade III after CCRT.

Glioma residual tumor volume is also an independent risk factor for cognitive impairment in postoperative patients with HGG after radiotherapy. The risk of cognitive impairment in patients with HGG volume greater than $5.58 \mathrm{~cm}^{3}$ after CCRT is greater than volume less than $5.58 \mathrm{~cm}^{3}$. Our findings are consistent with the previous study showing that tumor itself significantly affects cognitive functions, mainly due to the mass effect and higher grading [30].

Although this study provides important findings about risk factor on cognitive impairment of the postoperative patients with HGG after radiotherapy, limitations must be acknowledged. Firstly, we have only studied one molecular biological indicator, MGMT. Under the precise medical treatment model, more molecular biology and genetic indicators for the risk of cognitive impairment need to be further explored. Secondly, multicenters can affect the following issues which might cause unavoidable biases: (1) the TMZ manufacturer and the MGMT promoter methylation molecular biology testing company was not specified; (2) imaging examination may be influenced by the subjective judgment of the physician; (3) radiotherapy effects may be affected by the type of equipment and physician's skills.

Detailed classification of specific location of tumor did play a role in the study of cognitive impairment. Cox univariate analysis showed significant differences between different locations of tumors, among which tumors at temporal lobe manifested the most significant effect on cognitive impairment. Cox multivariable analysis showed no significant differences at different locations of tumor (Table 3).

In summary, cognitive impairment in postoperative patients with HGG after radiotherapy and chemotherapy is a significant clinical issue. MGMT promoter methylation, glioma WHO tumor grade and residual tumor volume are independent risk factors for cognitive impairment. We suggest patients at high risk should be closely monitored and provided with recommendations for appropriate interventions, individualized treatment and optimized patient management. Both clinical and genetic factors might simultaneously be associated with cognitive impairment developed during radiotherapy plus TMZ. Future studies require multi-center cooperation. More patients and more biological and genetic indicators in larger prospective studies should be included in the construction of more accurate and more effective risk prediction models.

\section{Conflicts of Interest}

Conflict of interest relevant to this article was not reported.

\section{References}

1. Ostrom QT, Gittleman H, Fulop J, Liu M, Blanda R, Kromer $\mathrm{C}$, et al. CBTRUS statistical report: primary brain and central nervous system tumors diagnosed in the United States in 20082012. Neuro Oncol. 2015;17 Suppl 4:iv1-62.

2. Klein M, Taphoorn MJ, Heimans JJ, van der Ploeg HM, Vandertop WP, Smit EF, et al. Neurobehavioral status and healthrelated quality of life in newly diagnosed high-grade glioma patients. J Clin Oncol. 2001;19:4037-47.

3. Tucha O, Smely C, Preier M, Lange KW. Cognitive deficits before treatment among patients with brain tumors. Neuro- surgery. 2000;47:324-33.

4. Sarkissian V. The sequelae of cranial irradiation on human cognition. Neurosci Lett. 2005;382:118-23.

5. Armstrong CL, Hunter JV, Ledakis GE, Cohen B, Tallent EM, Goldstein $\mathrm{BH}$, et al. Late cognitive and radiographic changes related to radiotherapy: initial prospective findings. Neurology. 2002;59:40-8.

6. Wang M, Cairncross G, Shaw E, Jenkins R, Scheithauer B, Brachman D, et al. Cognition and quality of life after chemotherapy plus radiotherapy (RT) vs. RT for pure and mixed 
anaplastic oligodendrogliomas: radiation therapy oncology group trial 9402. Int J Radiat Oncol Biol Phys. 2010;77:662-9.

7. Laack NN, Brown PD, Ivnik RJ, Furth AF, Ballman KV, Hammack JE, et al. Cognitive function after radiotherapy for supratentorial low-grade glioma: a North Central Cancer Treatment Group prospective study. Int J Radiat Oncol Biol Phys. 2005; 63:1175-83.

8. Klein M, Heimans JJ, Aaronson NK, van der Ploeg HM, Grit J, Muller M, et al. Effect of radiotherapy and other treatmentrelated factors on mid-term to long-term cognitive sequelae in low-grade gliomas: a comparative study. Lancet. 2002;360: 1361-8.

9. Verger E, Gil M, Yaya R, Vinolas N, Villa S, Pujol T, et al. Temozolomide and concomitant whole brain radiotherapy in patients with brain metastases: a phase II randomized trial. Int J Radiat Oncol Biol Phys. 2005;61:185-91.

10. Antonadou D, Paraskevaidis M, Sarris G, Coliarakis N, Economou I, Karageorgis P, et al. Phase II randomized trial of temozolomide and concurrent radiotherapy in patients with brain metastases. J Clin Oncol. 2002;20:3644-50.

11. Saito K, Mukasa A, Narita Y, Tabei Y, Shinoura N, Shibui S, et al. Toxicity and outcome of radiotherapy with concomitant and adjuvant temozolomide in elderly patients with glioblastoma: a retrospective study. Neurol Med Chir (Tokyo). 2014; 54:272-9.

12. Nasreddine ZS, Phillips NA, Bedirian V, Charbonneau S, Whitehead V, Collin I, et al. The Montreal Cognitive Assessment, MoCA: a brief screening tool for mild cognitive impairment. J Am Geriatr Soc. 2005;53:695-9.

13. Smith T, Gildeh N, Holmes C. The Montreal Cognitive Assessment: validity and utility in a memory clinic setting. Can J Psychiatry. 2007;52:329-32.

14. Wang Q, Qi F, Song X, Di J, Zhang L, Zhou Y, et al. A prospective longitudinal evaluation of cognition and depression in postoperative patients with high-grade glioma following radiotherapy and chemotherapy. J Cancer Res Ther. 2018; 14(Suppl):S1048-51.

15. Durand T, Jacob S, Lebouil L, Douzane H, Lestaevel P, Rahimian A, et al. EpiBrainRad: an epidemiologic study of the neurotoxicity induced by radiotherapy in high grade glioma patients. BMC Neurol. 2015;15:261.

16. Dhermain F. Radiotherapy of high-grade gliomas: current standards and new concepts, innovations in imaging and radiotherapy, and new therapeutic approaches. Chin J Cancer. 2014;33:16-24.

17. Kim BS, Seol HJ, Nam DH, Park CK, Kim IH, Kim TM, et al. Concurrent chemoradiotherapy with temozolomide followed by adjuvant temozolomide for newly diagnosed glioblastoma patients: a retrospective multicenter observation study in Korea. Cancer Res Treat. 2017;49:193-203.

18. Kole AJ, Park HS, Yeboa DN, Rutter CE, Corso CD, Aneja S, et al. Concurrent chemoradiotherapy versus radiotherapy alone for "biopsy-only" glioblastoma multiforme. Cancer. 2016;122:2364-70.

19. Woo PY, Ho JM, Tse TP, Lam SW, Mak CH, Chan DT, et al. Determining a cut-off residual tumor volume threshold for patients with newly diagnosed glioblastoma treated with temozolomide chemoradiotherapy: a multicenter cohort study. J Clin Neurosci. 2019;63:134-41.

20. Yang K, Jung SW, Shin H, Lim DH, Lee JI, Kong DS, et al. Cancer genetic markers according to radiotherapeutic response in patients with primary glioblastoma: radiogenomic approach for precision medicine. Radiother Oncol. 2019;131:66-74.

21. Wefel JS, Noll KR, Rao G, Cahill DP. Neurocognitive function varies by IDH1 genetic mutation status in patients with malignant glioma prior to surgical resection. Neuro Oncol. 2016;18: 1656-63.

22. Kesler SR, Noll K, Cahill DP, Rao G, Wefel JS. The effect of IDH1 mutation on the structural connectome in malignant astrocytoma. J Neurooncol. 2017;131:565-74.

23. Jiang $X$, Reardon DA, Desjardins A, Vredenburgh JJ, Quinn JA, Austin AD, et al. O6-methylguanine-DNA methyltransferase (MGMT) immunohistochemistry as a predictor of resistance to temozolomide in primary CNS lymphoma. J Neurooncol. 2013;114:135-40.

24. Binabaj MM, Bahrami A, ShahidSales S, Joodi M, Joudi Mashhad M, Hassanian SM, et al. The prognostic value of MGMT promoter methylation in glioblastoma: a meta-analysis of clinical trials. J Cell Physiol. 2018;233:378-86.

25. Smrdel U, Popovic M, Zwitter M, Bostjancic E, Zupan A, Kovac $\mathrm{V}$, et al. Long-term survival in glioblastoma: methyl guanine methyl transferase (MGMT) promoter methylation as independent favourable prognostic factor. Radiol Oncol. 2016;50:394-401.

26. Liu Y, Zhou R, Sulman EP, Scheurer ME, Boehling N, Armstrong GN, et al. Genetic modulation of neurocognitive function in glioma patients. Clin Cancer Res. 2015;21:3340-6.

27. Miotto EC, Junior AS, Silva CC, Cabrera HN, Machado MA, Benute GR, et al. Cognitive impairments in patients with low grade gliomas and high grade gliomas. Arq Neuropsiquiatr. 2011;69:596-601.

28. Noll KR, Sullaway C, Ziu M, Weinberg JS, Wefel JS. Relationships between tumor grade and neurocognitive functioning in patients with glioma of the left temporal lobe prior to surgical resection. Neuro Oncol. 2015;17:580-7.

29. Surma-aho O, Niemela M, Vilkki J, Kouri M, Brander A, Salonen $\mathrm{O}$, et al. Adverse long-term effects of brain radiotherapy in adult low-grade glioma patients. Neurology. 2001;56:128590.

30. Talacchi A, Santini B, Savazzi S, Gerosa M. Cognitive effects of tumour and surgical treatment in glioma patients. J Neurooncol. 2011;103:541-9. 